\title{
Percepción de adultos mayores chilenos en relación a la salud y el ejercicio físico en pandemia Covid-19 \\ Perception of Chilean older adults in relation to health and physical exercise in pandemic Covid-19
}

\author{
*Aquiles Alejandro Almonacid-Fierro, **Manuel Alberto Almonacid Fierro \\ *Universidad Católica del Maule (Chile), **Universidad Autónoma de Chile (Chile)
}

Resumen. El propósito de este artículo es comprender el significado que le otorgan adultos mayores de la zona central de Chile al periodo de la pandemia Covid-19 respecto a la salud y al ejercicio físico. Desde el punto de vista metodológico, la investigación se inscribe en la perspectiva del paradigma interpretativo-comprensivo, a través de metodología cualitativa. Se entrevistó a 17 adultos mayores en dos ocasiones, año 2020 año 2021, las entrevistas se realizaron vía Skipe o video llamada producto de las restricciones impuestas por la autoridad sanitaria, para evitar el contacto físico con los entrevistados. Los hallazgos del estudio dicen relación con los efectos adversos en términos psicosociales, que ha experimentado la población de adultos mayores producto del confinamiento, por otro lado, a pesar de la pandemia, los adultos mayores han mantenido hábitos saludables. En conclusión, se propone profundizar en estudios que permitan mostrar el impacto negativo del encierro en los adultos mayores y de esta manera influir para que el Estado asuma una mejor dirección en las políticas públicas dirigidas a este grupo etario.

Palabras clave: Adultos mayores, Covid-19; ejercicio físico, calidad de vida.

\begin{abstract}
The purpose of this article is to understand the meaning given by elderly adults in central Chile to the period of the Covid-19 pandemic concerning health and physical exercise. From the methodological perspective, the research is inscribed in the perspective of the interpretative-comprehensive paradigm, through the qualitative methodology. Seventeen seniors were interviewed on two occasions; the year 2020, the year 2021, the interviews were performed via Skype or video call due to the restrictions imposed by the sanitary authority, to avoid physical contact with the interviewees. The results of the study are related to the adverse effects in psychosocial terms that the elderly population has experienced as a result of the confinement, oppositely, despite the pandemic, the seniors have maintained healthy lifestyles. In conclusion, this study proposes further studies to show the negative impact of confinement on elderly adults and, as follows, influence the State to assume a better direction in public policies aimed at this age group.
\end{abstract}

Key words: Elderly adults, Covid-19; physical exercise, quality of life.

\section{Introducción}

La expansión de la población de edad avanzada ha alcanzado cifras históricas y tiende a aumentar aún más en los próximos decenios. Para 2050, una de cada seis personas en el mundo tendrá más de 65 años, en comparación con una de cada 11 en 2019. Para 2050, una de cada cuatro personas viviendo en Europa y América del Norte podría tener 65 años y más (Beard et al., 2016; WHO, 2015). Según datos del Instituto Nacional de Estadísticas (INE) Chile presenta el año 2020 un indicador de esperanza de vida alto, con 77,4 años para los hombres y 82,2 para las mujeres, alcanzando al final del período proyectado, es decir en 2050, a 83,2 años para los

Fecha recepción: 27-05-21. Fecha de aceptación: 20-09-21

Aquiles Alejandro Almonacid-Fierro

aalmonacid@ucm.cl hombres y 87,8 años para las mujeres, lo que representará el 25\% de la población en el año 2050 (INE, 2018). A pesar del envejecimiento de la población, desafortunadamente hay poca visibilidad y apreciación de esta porción de la población (Condeza et al., 2016).

En los últimos meses el mundo ha sido testigo de la evolución de una pandemia infecciosa llamada Enfermedad del Coronavirus (Covid-19) cuyo agente etiológico es el coronavirus del síndrome respiratorio agudo severo el SARS-CoV-2 (WHO, 2020; Crespo \& Crespo, 2020). El Covid-19 se ha definido como un conjunto de enfermedades respiratorias agudas, que se transmite de persona a persona a través del contacto con secreciones infectadas, principalmente a través del contacto con gotitas respiratorias grandes (Bonanad et al., 2020; Maguiña-Vargas, Gastelo \&Tequen, 2020; Williams \& Cañon-Montañez, 2020). Los adultos mayores se destacan en la pandemia de Covid-19, en gran parte debi- 
do a los cambios resultantes de la senescencia o la senilidad (Sannicandro, Cofano, Rosa \& Colella, 2020; Applegate \& Ouslander, 2020). La pandemia de Covid19 ha puesto de relieve a los adultos mayores, principalmente debido al potencial de riesgo de este grupo, con acciones y estrategias de distanciamiento social, dirigidas específicamente a este grupo (Brooke \& Jackson, 2020). Las acciones para proteger a los adultos mayores en la pandemia incluyeron la estratificación por edad, que a pesar de ser positiva como organización del servicio, reforzó los prejuicios de la sociedad y la sobrevaloración de características eminentemente negativas de las personas mayores (Armitage \& Nellums, 2020; Berg-Weger \& Morley, 2020).

El perfil epidemiológico de la población de edad avanzada se caracteriza por la triple carga de enfermedades con un fuerte predominio de las afecciones crónicas, la prevalencia de una alta mortalidad y morbilidad por causas externas agudas y las afecciones crónicas agudas, ya que la mayoría de los adulto mayores son portadores de enfermedades o disfunciones orgánicas (Bonsdorff \& Rantanen, 2011; Conde-Sala; Portellano-Ortiz, CalvóPerxas, \& Garre-Olmo, 2017; Fernández-Ballesteros, 2011). Sin embargo, es importante destacar que este panorama no significa necesariamente una limitación de sus actividades, una restricción de la participación social o del desempeño de su función social, que actualmente está sufriendo un gran impacto debido a las medidas de control producto de la pandemia, en el que se ha recomendado el aislamiento social como principal medio de prevención de la enfermedad de Covid-19 (Ayalon et al., 2020; Bonanad et al., 2020; Grossman, Hoffman, Palgi, \& Shrira, 2020). Para comprender las repercusiones psicológicas y psiquiátricas de una pandemia, como en el caso de Covid-19, algunos grupos específicos son especialmente vulnerables, como los adultos mayores, los inmunodeprimidos, los pacientes con afecciones clínicas y psiquiátricas previas, los familiares de pacientes infectados y los residentes en zonas de alta incidencia, tal como lo presentan los estudios de Cuello-Carballo et al. (2020); Pérez Abreu, Gómez Tejeda, \& Dieguez Guach, (2020); Rahman \& Jahan, (2020).

Para envejecer con éxito, es necesario que, además de estimular el buen funcionamiento físico y mental, estén presentes las actividades sociales (PinazoHernandis, 2020; Tavares et al., 2017). Varios estudios han planteado que las personas mayores tienden a asociar su calidad de vida con los siguientes aspectos: la práctica de ejercicio físico, el acceso al ocio, la realiza- ción de actividades intelectuales y, en particular, la convivencia con la familia, la comunidad y la sociedad (Netuveli, \& Blane, 2008; Forte, Boreham, De Vito \& Pesce, 2015; Li et al., 2014). Por otro lado, las relaciones interpersonales juegan un papel extremadamente beneficioso en la calidad de vida para los adultos mayores, tanto los lazos familiares como las viejas relaciones, así como esas nuevas amistades construidas en los grupos comunitarios. Teniendo en cuenta que los adultos mayores son la población en la que el índice de mortalidad por el Covid-19 es más alto, es importante y sumamente necesario evaluar el impacto del virus en la calidad de vida de la población anciana (Ping et al. 2020).

La calidad de vida de las personas mayores está relacionada con la autonomía de las actividades diarias y de ocio, la satisfacción de los deseos presentes y futuros, la interacción social, y lo bien que se está mentalmente consigo mismo (Vanleerberghe et al., 2017; Chou, Hwang, \& Wu, 2012; Villarreal-Angeles, MoncadaJimenez \& Ruiz-Juan, 2021). Al referirse a la calidad de vida de los adultos mayores, el concepto adquiere mayor visibilidad ya que, para esta parte de la población, la vida no puede ser percibida como una dificultad, sino que requiere construcciones que presenten nuevas posibilidades de descubrimiento y superación, con el objetivo de alcanzar un estado de felicidad personal (Li et al., 2014; Netuveli \& Blane, 2008; Tavares et al., 2017). Por lo tanto, la calidad de vida en la vejez implica un panorama complejo, ya que abarca aspectos objetivos y subjetivos de la vida de los adultos mayores. Los factores objetivos se refieren a la ausencia de enfermedad y a la capacidad funcional, centrándose en los aspectos biológicos y epidemiológicos, mientras que los factores subjetivos se refieren a la propia comprensión del individuo de los valores que posee, sus expectativas, objetivos y preocupaciones (Anton et al., 2015; Conde-Sala et al., 2017; Farajzadeh, Gheshlagh \& Sayehmiri, 2017).

La capacidad funcional se define como el rendimiento para la realización de actividades cotidianas o de la vida diaria (Alcañiz \& González-Moro, 2020). La práctica de la actividad física también promueve la mejora de la composición corporal, la reducción del dolor articular, el aumento de la densidad mineral ósea, la mejora del uso de la glucosa, la mejora del perfil lipídico, el aumento de la capacidad aeróbica, la mejora de la fuerza y la flexibilidad, la reducción de la resistencia vascular (De Carvalho et al., 2017; De Souto Barreto et al., 2016; Hale \& Marshall, 2017; Heredia, Rodríguez \& García, 2021; Vicentini de Oliveira et al., 2021). El tipo de ejercicio físico recomendado para los adultos mayo- 
res en el pasado era más aeróbico debido a sus efectos sobre el sistema cardiovascular y el control de estas enfermedades, además de los beneficios psicológicos (Issa, Kilpi \& Webber, 2016; Whitehead \& Blaxton, 2017). La práctica regular de ejercicios físicos promueve una mejora fisiológica, ayuda al control de la glucosa, mejor calidad del sueño, mejora de la capacidad física relacionada con la salud, una mejora psicológica, relajación, reducción de los niveles de ansiedad y estrés, mejora del estado de ánimo, mejoras cognitivas y una mejora social, produce individuos más seguros, mejor integración social y cultural, integración comunitaria, ampliación de la red social y cultural, entre otros, además de la reducción o prevención de algunas enfermedades como la osteoporosis y las desviaciones posturales, tal como lo reporta la literatura en los estudios de Chou et al.(2012); Dunsky, (2019); Forte et al., 2015; GarciaMolina; Carbonell-Baeza \& Delgado-Fernández, (2010); Bonsdorff \& Rantanen, (2011); Huxhold, Miche \& Schüz, (2013); Russo et al., 2020; Torres, Gaibor, \& Pozo (2020); Viladrosa, Casanova, Ghiorghies \& Jürschik, (2017).

La actividad física también mejora el bienestar psicológico en cuanto a la relajación y la reducción de los niveles de ansiedad y estrés, la mejora del estado de ánimo, las mejoras cognitivas y el bienestar social, lo que lleva a los mayores a conseguir múltiples beneficios para su salud, a sentirse más seguros, más cohesionados socialmente e integrados en sus respectivas comunidades (Li et al., 2014). En los adultos mayores, la práctica de ejercicio físico regular reduce el riesgo de caídas y previene o retrasa la aparición de enfermedades crónicas asociadas al envejecimiento. Además de los beneficios ya mencionados, la práctica de actividad física en las personas mayores tiene un efecto favorable en el aumento del consumo de oxígeno, la mejora del control glucémico, la reducción de dolencias, el aumento de la tasa metabólica basal, la mejora del equilibrio y la marcha, la reducción del riesgo de caídas y fracturas (Tyndall, Clark \& Anderson, 2018, Froment \& González, 2018; Heredia et al., 2021)

En este contexto, estudiar cómo la pandemia Covid19 ha afectado a las personas mayores en su calidad de vida y en la práctica regular de ejercicio físico se torna todo un desafío, toda vez, que son factores determinantes para la mantención de un estado de salud activo y saludable. En consecuencia, el objetivo del presente estudio es conocer y comprender la percepción de las personas mayores respecto al ejercicio físico y la salud, en tiempos de pandemia Covid-19, en el bien entendido que ha resultado un tiempo difícil y complejo, en particular para los adultos mayores.

\section{Material y método}

La presente investigación es un estudio bajo el paradigma interpretativo (Flick, 2018), en el que se pretende indagar sobre la percepción de las personas mayores, respecto al ejercicio físico y la salud en tiempos de pandemia Covid-19. El enfoque metodológico y analítico del estudio está vinculado al campo de la investigación cualitativa, puesto que a través de la investigación cualitativa el investigador puede comprender los sentimientos, creencias, valores, recuerdos y experiencias de los sujetos lo que corresponde a un espacio más profundo de relaciones, procesos y fenómenos (Given, 2008; Moreira \& Costa, 2016). Se realizaron 17 entrevistas semiestructuradas a personas mayores considerando los siguientes criterios de inclusión: mujeres y hombres mayores de 65 años, interés en participar en el estudio, firma de consentimiento informado; haber participado el año 2019 en un taller deportivo-recreativo financiado por el Instituto Nacional de Deportes (IND) en la región del Maule, Chile; haberse matriculado el año 2020 en el referido taller y personas autovalentes física y cognitivamente. Como criterios de exclusión se determinó una edad inferior a 65 años y no haber participado del programa para adultos mayores del IND el año 2019.

Los datos del estudio fueron recogidos durante el año 2020, en el periodo julio- septiembre y seguidamente durante los meses de febrero y marzo del año 2021, en que se realizó una segunda entrevista a 10 de los participantes del año 2020, con la idea de profundizar en algunos aspectos asociados a su calidad de vida, luego de vivir un año de pandemia. El instrumento utilizado fue un guion de entrevista, que fue elaborado por el equipo investigador, en función de las dimensiones del problema en estudio. El guion fue validado por cinco académicos expertos, quienes, a través de una revisión exhaustiva, contribuyeron a que las interrogantes fueran específicas y se focalizarán en los objetivos planteados. Algunas de las preguntas que se hicieron a los participantes se presentan a continuación: ¿Cómo ha impactado la pandemia Covid-19 en su calidad de vida y en la realización de actividad física?; ¿Que ejercicios físicos ha realizado durante la pandemia y con qué estrategias ha cuidado su salud?; Cómo ha cambiado su vida producto de la pandemia Covid-19, en términos familiares, sociales, psicológicos y de salud en general?; ¿Qué tipo de ejercicios para mantener su salud ha rea- 
lizado en casa durante la pandemia?; ¿Qué tipo de apoyo a tenido de sus familiares para mantener actividad física y hábitos de saludables?

Antes de la recopilación de datos, los investigadores obtuvieron un consentimiento informado por escrito para garantizar la confidencialidad de los nombres de las personas, teniendo en cuenta la privacidad y haciendo hincapié en la participación voluntaria. Se informó a los participantes sobre los propósitos de la investigación y se les solicitó su autorización para grabar la entrevista con el propósito de salvaguardar los aspectos éticos del estudio de acuerdo a la declaración de Helsinki. La entrevista se realizó vía skipe y video llamada, debido a la emergencia sanitaria por la que atraviesa el país y tuvo una duración promedio de 60 minutos.

Para el tratamiento de los datos se ha optado por un análisis de contenido (Bengtsson, 2016; Graneheim, Lindgren \& Lundman, 2017), que comprende tres flujos de actividad: la condensación de los datos, la presentación de los datos y la elaboración/verificación de conclusiones. Cabe señalar, que las dimensiones se obtuvieron a partir de la revisión del marco teórico (Gibbs, 2007). En consecuencia, traemos estos fragmentos de los datos y los reunimos para crear categorías, que definimos con base en alguna propiedad. Posteriormente se agrupan las categorías previas a través de la codificación abierta, cuestión que permite la emergencia de las categorías primarias, dando paso desde un primer momento descriptivo, a una instancia de carácter interpretativo (Flores, 2009).

Los datos se analizaron utilizando la lógica inductiva de categorización teórica, la que se basa en la categorización como la principal herramienta analítica de datos, destinada a teorizar a través de operaciones que conducen a la construcción teórica (Morse, 2015; Silverman, 2014;Vaismoradi, Turunen, \& Bondas, 2013), este proceso fue asistido por el programa Nvivo 10. El equipo de investigación condujo esta etapa en la secuencia siguiente: i) Los datos fueron revisados en su totalidad de manera abierta, intentado responder a la pregunta ¿qué nos dicen los datos? Las informaciones levantadas se reagruparon en seis categorías clasificatorias que posteriormente fueron evolucionando con las siguientes fases de la codificación; ii) En una segunda fase, se establecieron conexiones entre los códigos con el fin de construir categorías descriptivas y explicativas. Estas categorías serán detalladas en la sección de resultados de este artículo; iii) En la última fase, corresponde a la elaboración teórica, cuya finalidad es producir un marco explicativo que permita comprender las parti- cularidades que las personas mayores otorgan al ejercicio físico y la salud en tiempos de pandemia Covid-19, así como también explicar las articulaciones de aspectos asociados a la calidad de vida.

\section{Resultados}

A continuación, se presenta la matriz, cuya mega categoría se centra en la percepción de la salud en personas mayores en tiempos de Covid-19. Del análisis de los datos emergen dos categorías primarias, a saber: Impacto del Covid-19 en la calidad de vida y Ejercicio en Tiempos de Covid-19, y seis categorías secundarias que se presentan según la codificación realizada a los documentos primarios. Cada relato presentado posee un código que expresa la siguiente nomenclatura: sujeto y número de entrevista.

Tabla 1.

Matriz de sistematización de los datos y descriptores

\begin{tabular}{|c|c|c|c|}
\hline Categoría & Categoría primaria & Descriptor & Categoría secundaria \\
\hline $\begin{array}{l}\text { Percepción } \\
\text { de salud en } \\
\text { Personas }\end{array}$ & $\begin{array}{l}\text { Impacto del Covid- } \\
19 \text { en la calidad de } \\
\text { vida }\end{array}$ & $\begin{array}{l}\text {-Categoría que alude al impacto de } \\
\text { la pandemia en la calidad de vida } \\
\text { de las personas mayores. }\end{array}$ & $\begin{array}{l}\text { le Estado funcional } \\
\text { Estado psicosocial } \\
\text { Necesidad de socialización }\end{array}$ \\
\hline $\begin{array}{l}\text { Mayores en } \\
\text { Tiempos de } \\
\text { Covid-19 }\end{array}$ & $\begin{array}{l}\text { Ejercicio físico en } \\
\text { tiempos de covid- } \\
19\end{array}$ & $\begin{array}{l}\text { Categoría que aborda la práctica } \\
\text { de actividad física de personas } \\
\text { mayores en tiempos de pandemia }\end{array}$ & $\begin{array}{l}\text { Hábitos saludables } \\
\text { Tipología de ejercicios } \\
\text { Apoyo familiar }\end{array}$ \\
\hline
\end{tabular}

1.- Categoría primaria: Impacto del Covid19 en la calidad de vida - Categoría Secundaria: Estado Funcional

El estado funcional del Adulto Mayor refiere a la capacidad de la persona para realizar las actividades de la vida diaria, sin necesidad de supervisión, dirección o asistencia, comprometiendo habilidades físicas, mentales y sociales al ejecutar tareas y desempeñar roles sociales en su medio. Sobre esto, las personas mayores entrevistadas plantean lo que sigue:

"He perdido toda la movilidad que tenía antes de la pandemia, ese es el principal impacto, porque el ejercicio físico me ayuda a tener mejor movilidad y mejora la capacidad para hacer las cosas en casa» (S-13).

«Soy autovalente hago todas mis cosas sola, no he visitado al médico para evitar contacto con otras personas, tomo medicamentos para la hipertensión hace 20 años, los compro en la farmacia, salgo a caminar dos veces por semana y me hace bien. (S-1)

De acuerdo a los relatos de los informantes clave, estos refieren un buen grado de adaptación a las condiciones restrictivas de la pandemia, en general muestran un estado funcional acorde a las necesidades y requerimientos de sus actividades diarias, lo cual puede explicarse por la ruralidad que presenta la región del Maule en Chile, que obliga al individuo a realizar dia- 
riamente una buena cantidad de acciones físicas.

"Todo lo puedo hacer sola, no tengo problemas físicos, veo mi jardín, mis gallinas, hago el aseo en casa, no he ido al médico, solo recibo medicamentos que me manda el Centro de Salud Familiar (CESFAM) de Maule, para la presión y el colesterol.» (S-7)

"Para hacer las cosas habituales, compras, el aseo en casa no tengo problemas, estado emocional perfecto, solo tenemos contacto telefónico con la familia, tengo dolores típicos de viejo, una pequeña artrosis en la rodilla derecha, no he tenido consulta con médico hace 2 años, el 6 de mayo tengo examen de sangre, me lo mandó por teléfono el doctor, casi no hago ejercicio menos ahora que llegó el invierno» ( $S$-10)

Las personas entrevistas relatan que se mantienen ocupados, refieren además uso de medicamentos que les ayudan a sobrellevar de mejor forma sus actividades diarias, mejorando la funcionalidad. Por otro lado, manifiestan que realizan con relativa normalidad las labores de casa, y que se mantienen con los medicamentos y los exámenes que se realizan en los Centros de Salud.

\section{Categoría primaria: Impacto del Covid-19 en la calidad de vida - Categoría secundaria: Estado Psicosocial}

Los adultos mayores en estado de fragilidad recibieron la cuarentena como una medida de protección, pero los autovalentes y sanos, lo sufrieron como un castigo, provocando distintas sensaciones como las reportadas aquí:

"Me siento insegura y asustada, la gente es muy irresponsable, la gente no se cuida. Los familiares no me pueden venir a ver, solo nos comunicamos por teléfono, extrañamos poder ver a la familia, tengo mucho respeto y temor» (S-3).

"La pandemia me tomó por sorpresa igual que a todo el mundo, y a mí me impacto esto de estar encerrada, de no hacer cosas, tener mucho miedo, un miedo a salir, a que te contagien y un sinfín de cosas que pasan, pero más que nada es el miedo.» $(S-5)$.

Las cuarentenas decretadas por el Gobierno afectaron mayormente a los adultos mayores, quienes representan más del 60 por ciento de los casos de fallecidos por el virus y si consideramos que alrededor del 30 ciento de los adultos mayores viven solos o acompañados de otro adulto mayor, el confinamiento al que se les sometió provocó un aislamiento social que les dificultaba incluso poder acceder a alimentos o medicamentos

"Nos tienen encerradas todas aquí estresadas y la gente poco se cuida, por culpa de algunos irresponsables nosotros seguimos encerrados todavía. El encierro a uno la estresa un poco, se siente frustración por no poder salir porque uno está ence- rrado no puede practicar deporte (S-6)

"Nos ha aislado de todo, de la familia, del deporte, sin poder salir, la pandemia y el confinamiento produjo mucho daño, hemos perdido la libertad, tenemos que estar cumpliendo órdenes día a día, claro en beneficio personal pero igual nos afecta, yo extraño a las compañeras, con quienes nos juntábamos, entonces el tiempo se nos hace infinito.» (S-8).

Los adultos mayores entrevistados refieren sentirse asustados, inseguros, con miedo al contagio, por otro lado, algunos relatan sentirse estresados y frustrados por no poder salir, por perder su libertad, se sienten aislados física y emocionalmente, extrañan el contacto y la socialización

3. Categoría primaria: Impacto del Covid-19 en la calidad de vida - Categoría Secundaria: Necesidad de socialización

Los adultos mayores son capaces de analizar el fenómeno de las cuarentenas con espíritu crítico y se permiten hacer aportes, establecer sugerencias respecto a la mejora de sus condiciones de vida, tal como se representa a continuación.

"Me hace falta salir a vitrinear al centro de la ciudad, tomar un café, conversar con amigos..., nos reunimos por Zoom, hablo por video llamada con mis hijos que viven en Santiago, pero no es lo mismo, quisiera sus abrazos» $(S-1)$.

"Me gustaría que pudiéramos juntarnos nuevamente con el grupo de Adultos Mayores para hacer ejercicio en el centro comunitario, por otro lado, del Municipio viene una persona y nos da tareas una vez por semana, para tener la mente ocupada.» (S-3)

De acuerdo a los relatos precedentes, podemos encontrar diversas reacciones y propuestas, referidas principalmente a facilitar el retorno a las actividades cotidianas, los sujetos desean volver a encontrarse con sus amistades, sentir que alguien se preocupa por ellos. Lo anterior, toda vez que la necesidad de socialización para este grupo etario es fundamental, puesto que les permite vivir una vejes más plena.

"Me gusta que se preocupen de nosotros, el CESFAM nos llama, nos pregunta que necesitamos, la kinesióloga y la terapeuta... Hicieron visita a las casas. Doy gracias a Dios que puedo caminar y que tengo condiciones favorables por vivir en el campo.» $(S-7)$.

"Los locales donde uno hace práctica de ejercicio son muy pocos, cuesta conseguir hora, porque les dan preferencia a otros grupos, los grupos de Adulto Mayor tienen poca prioridad, a horas poco apropiadas, nos dan hora muy temprano, es como hacer deporte desde las 5 de la mañana y después dicen que "hay que cuidar a los adultos mayores». Lo otro es hacer más publicidad para las actividades deportivas y recreativas.» (S- 
10)

Los participantes de la investigación, en sus relatos, reportan que los factores psicológicos y emocionales son relevantes a la hora de evaluar su calidad de vida, y en este sentido, revelan una crítica a las medidas parciales del gobierno y a la falta de priorización de los en las políticas de cuidado para los adultos mayores. Lo anterior, se manifiesta cuando por ejemplo señalan que no cuentan con ligares para la práctica de actividad física.

4.- Categoría primaria: Ejercicio físico en tiempos de covid-19 - Categoría Secundaria: Hábitos saludables

El mantenimiento de hábitos saludables cumple un propósito de prevención, permitiendo que, al llegar a la tercera edad, las personas puedan mantener sus funciones el mayor tiempo posible, es así que nuestros informantes expresan lo siguiente:

"Es bueno mantener la actividad física y comer bien, se puede, a mí no me ha afectado la pandemia en ese sentido, ya que he podido hacer mi vida normal y alimentarme naturalmente.» (S-2).

"Cuesta más mantener hábitos saludables y es más difícil, por ejemplo, antes íbamos dos veces por semana al centro comunitario a las clases y el profesor nos hacía diferentes ejercicios, baile, yoga, pilates, todo eso debíamos hacer.» (S-3).

Los sujetos en este estudio otorgan una importancia relevante al ejercicio físico como un hábito saludable a mantener en el tiempo, mejorando con ello su calidad de vida, al reconocerse autovalentes, tal como lo expresan sus relatos. Reconocen por otro lado que la falta de ejercicio disminuye sus posibilidades en pandemia.

"No puedo realizar ninguna actividad física normal, porque todo era grupal, entonces como ahora es individual cuesta un poco más, éramos muchos los que íbamos.» (S-4).

"Estoy bien no me ha afectado tanto la pandemia, somos dos viejos ermitaños, salgo al patio, camino, hago elongaciones, hago elíptica, lo hago casi todos los días 10 minutos, mi señora hace todos los días casi 20 minutos» (S-10)

En menor medida le otorgan importancia a la alimentación y al acompañamiento. Por otro lado, reconocen que la pandemia ha afectado sus rutinas en todo nivel, entre estos hábitos se cuentan la actividad física, evitar consumo o exposición a cigarrillos, higiene, alimentación saludable, actividad social y acompañamiento afectivo

\section{5.- Categoría primaria: Ejercicio físico en} tiempos de covid-19 - Categoría Secundaria: Tipología de ejercicios

Los adultos mayores consultados mencionan algunos ejercicios ejecutados en Pandemia, lo que permite constatar que existe conciencia en las personas sobre los beneficios del ejercicio regular Los sujetos investigados relatan lo siguiente respecto a los tipos de ejercicios:

"Siempre nos recalcaban en el taller al que iba lo de caminar, incluso antes de la pandemia, la idea era mantener las caminatas mantener una actividad física y nos ha costado eso de no salir para mantenerse protegidos» (S-4).

"Subo y bajo escaleras, bailo con mi nieta, tengo una pieza donde hago ejercicios, hago abdominales en la cama, también puedo bailar, escuchar música, me encanta escuchar música, esas son las cosas que hago en el encierro.» (S-5)

En el relato de las personas mayores entrevistadas se observa una adherencia a la práctica regular, lo que se demuestra en los relatos al señalar que practican caminata, baile, ejercicios localizados. El ejercicio y la actividad física benefician a los adultos mayores. Las actividades de resistencia, o aeróbicas, aumentan la capacidad aeróbica. Algunos ejemplos son las caminatas o correr, bailar, nadar y andar en bicicleta.

«Bailar, hacer ejercicios con banda, con un palito (bastones) y aquí yo uso un pedazo de PVC para levantar los brazos, mover el bastón de un lado a otro o con una banda elástica también se pisa con el pie y se levanta el brazo, se puede abrazar un poste con la banda y se hacen los ejercicios de brazos, todos esos ejercicios los hacíamos con el profesor en el taller.» (S-8)

«Me gustaba salir a caminar al parque, pero durante la pandemia no he podido realizar las caminatas, ya que el encierro no nos deja, pero realizamos caminatas dentro de la casa.» (S.14).

De acuerdo al relato de los entrevistados, los ejercicios de fuerza mejoran la masa muscular y la densidad ósea. Levantar pesas o usar bandas elásticas pueden fortalecerle. Los ejercicios le estabilizan y ayudan a prevenir caídas. Los ejercicios de flexibilidad mejoran la movilidad de los músculos y pueden ayudar a que su cuerpo permanezca relajado.

6.- Categoría primaria: Impacto del Covid19 en la calidad de vida - Categoría Secundaria: Apoyo familiar

Para los adultos mayores entrevistados la familia tiene un lugar importante en su vida de relación y en ocasiones no se reconoce como familia solamente con quienes se comparte la consanguineidad, sino también a aquellos con quienes se vive en un momento determinado de su vida, los siguientes relatos dan cuenta de aquello:

"He caminado con una nieta de 26 años como una hora al menos tres veces por semana, antes de cuarentena y en cuarentena no he salido porque estuve enferma de vértigo, y es peligroso que salga a caminar sin compañía.» (S-5). 
"Solo he practicado en la casa, con mi esposo salimos a caminar todos los días media hora a lo menos, casi nadie nos visita, así que no tengo apoyo para hacer ejercicio, aunque mis hijos me llaman casi todos los días» (S-7).

Los sujetos de este estudio plantean que, si bien han realizado actividad física acompañados de familiares, en tiempos de cuarentena se han encontrado aislados de ellos, realizando actividades solo con familiares directos (esposo o esposa). Las enfermedades propias de la vejez conspiran en contra del desarrollo de la actividad física diaria, la compañía del esposo o esposa es vital, ya que puede ser la única compañía de que puedan disfrutar en tiempos de Covid-19.

"Mi Marido me acompaña a caminar casi día por medio, jardineamos, cortamos leña, todos los días nos mandan ejercicios para AM desde el Municipio CESFAM, nos mandan videos por WhatsApp de la Universidad, ejercicios para la pelvis. Vivimos solos y nadie nos puede acompañar por las cuarentenas.» (S-8).

"Solo salgo a caminar tres veces por semana, salgo sola no tengo apoyo de nadie, a veces veo videos de YouTube y hago ejercicios una vez a la semana.» (S-1).

Para las personas mayores participantes del estudio, la familia, puede ayudarlos a superar diversas situaciones de riesgo, como por ejemplo una enfermedad o un accidente y también pueden ayudarles a sentirse importantes, queridos, apoyarles en sus tareas diarias o simplemente acompañarles a realizar actividades rutinarias en medio de una pandemia, tal como se presenta en los relatos.

\section{Discusión}

Los resultados de la investigación mostraron que la percepción de las personas mayores sobre la salud y el ejercicio físico en la vejez están influenciadas por diferentes factores. El análisis de los datos reveló dos categorías y seis subcategorías, que se configuraron de la siguiente manera: la categoría «Calidad deVida» consideró los temas asociados al estado funcional de los adultos mayores, impacto de la cuarentena y sugerencias/requerimientos. Por su parte, la categoría «Ejercicio Físico» consideró aspectos asociados a hábitos saludables, tipos de ejercicios y apoyo familiar.

La literatura reporta que la pandemia ha generado un torbellino de emociones ligadas a la desesperanza, aburrimiento, soledad y depresión debido al aislamiento y, por otro lado, enojo, frustración o irritabilidad por la pérdida de autonomía y libertad personal, miedo y en ocasiones mucha ansiedad (Grossman et al., 2020; Viana, de Lima Silva \& de Lima, 2020; Van Tilburg et al., 2020). Sin embargo, a pesar de las consecuencias negativas que ha causado la pandemia de Covid-19 en los adultos mayores, hay que destacar que es en tiempos de crisis cuando el ser humano es resistente, y actúa con altruismo y cooperación. En consecuencia, para superar estas adversidades existenciales, es posible recurrir a pasatiempos, ejercicios físicos, lecturas, películas, meditaciones, oraciones, prácticas amorosas, labores domésticas, entre otras actividades, tal como lo presentan los relatos de los sujetos investigativos en el presente estudio y que son coincidentes con lo reportado por la literatura en los trabajos de Flett \& Heisel, (2020); Heredia et al. (2021); Losada-Baltar et al . (2020); Ornell, Schuch, Sordi \& Kessler, (2020); Pinazo-Hernandis, (2020). Para los adultos mayores, el aislamiento producto de las prolongadas cuarentenas puede ser más doloroso, debilitando su capacidad de adaptación y reacción, produciendo respuestas emocionales como los sentimientos de soledad, que repercuten en el estado de equilibrio mental, como se evidencia en los relatos de los sujetos entrevistados cuando señalan que la pandemia y el subsecuente confinamiento les ha hecho mucho daño a sus vidas.

Los resultados de este estudio están alineados con las últimas investigaciones en el área en cuanto a los beneficios del ejercicio físico en las personas mayores como resultado de mantener hábitos saludables y un nivel de actividad física adecuado (Beard et al., 2016; Farajzadeh et al.,2017; Hale \& Marshall, 2017; TeixeiraSalmela, 2016). El análisis de los datos de la investigación permite afirmar que la práctica regular de ejercicio físico en las personas mayores promueve el bienestar fisiológico en aspectos como el control de la glucosa, la mejor calidad del sueño, la mejora de la capacidad física relacionada con la salud, la reducción o prevención de algunas patologías como la osteoporosis y las desviaciones posturales y la mejora de la fuerza muscular (Chou et al., 2012; Garcia-Molina et al., 2010; Heredia et al., 2021; Ruaro et al., 2019). En este sentido, el ejercicio físico sistemático en tiempos de pandemia Covid-19 contribuye a un envejecimiento saludable de acuerdo a como lo expresan los sujetos participes del estudio, toda vez que la pandemia les ha permitido revalorar la práctica de actividad física y el impacto positivo en su salud y calidad de vida.

Entre otros resultados de la investigación, los adultos mayores informan que la actividad física mejora su calidad de vida, cuando realizan diversos tipos de ejercicio y mantienen hábitos saludables cuestión que es coincidente con lo reportado por De Oliveira, Vinhas 
\& Rabello, (2020); Faustino \& Neves, (2020). Si bien un reciente manual del American College of Sports Medicine recomienda un estilo de vida con actividades de intensidad ligera a moderada para optimizar la salud, estas mismas intensidades pueden ser necesarias para promover adaptaciones en el sistema cardiovascular y reducir los factores de riesgo de enfermedades cardiovasculares (Issa et al., 2016). Así, iniciar y mantener un programa de actividad física de intensidad leve a moderada durante un largo periodo de tiempo en personas mayores puede disminuir la tasa de declive relacionada con la edad en muchas funciones fisiológicas, lo que en última instancia beneficia la calidad de vida (De Souto-Barreto et al., 2016; Machón et al., 2017)

Dentro de la subcategoría «apoyo familiar», los participantes en el estudio relatan aquellos aspectos que les impiden, en ocasiones, una práctica sistemática de ejercicio físico, como por ejemplo estar acompañados por algunos miembros de la familia. En este sentido, los estudios sobre el envejecimiento indican que los adultos mayores lo ven como un proceso de pérdida en diversos ámbitos de la vida y la pérdida de relaciones sociales, situación que atribuyen a las dificultades de la edad, cuestiones que se han acrecentado producto del impacto psicosocial de la pandemia Covid-19 en la vida a de las personas mayores tal como lo reportan los estudios de Armitage \& Nellums, (2020); Berg-Weger \& Morley, (2020); Brooke \& Jackson, (2020); Grossman et al., 2020). Ante este panorama, cabe destacar que las personas mayores son capaces de redescubrir el valor de la vida en pareja y del ejercicio acompañado para superar las dificultades propias de la edad.

\section{Conclusiones}

El impacto del Covid-19 en personas mayores de 60 años que han pasado por experiencias traumáticas, de alguna manera, se relativizó en la crisis actual. Al evaluar los niveles de actividad física y aspectos generales de salud producto del aislamiento social, los adultos mayores declararon asumir la pandemia con dignidad y espíritu de sacrificio. En este escenario se observó que la soledad es una de las quejas más frecuentes, seguida de las enfermedades emocionales y psicológicas causadas por la distancia o la pérdida de familiares, la impotencia, el miedo a la muerte o incluso la negligencia de los familiares hacia los adultos mayores. Sin embargo, en el contexto social, es importante destacar la relación del Covid-19 y el distanciamiento social, así como la incidencia de los prejuicios generalizados sobre la edad, la estigmatización senil, la relación causal entre el aislamiento y la soledad, y las implicaciones para el comportamiento. Se observó que, en las personas mayores que realizaban actividad física con frecuencia durante la semana, se redujo la sensación de olvido y la distancia con las personas de su convivencia. En este punto, el contacto virtual puede no ser cualitativamente igual que el contacto cara a cara, pero es un facilitador de la unión social en tiempos de pandemia.

El envejecimiento de la población, es uno de los mayores desafíos de la salud pública contemporánea, constatándose este fenómeno en los países en desarrollo, como el caso de Chile, donde el envejecimiento de la población se ha producido de manera más acentuada en los últimos 20 años. En este sentido, podemos señalar que la necesidad de describir y analizar el impacto en la calidad de vida de las personas mayores en tiempos de la pandemia Covid-19 ha sido el propósito del presente estudio, considerando además los numerosos beneficios de la práctica regular de actividad física, que contribuye a mantener y mejorar la calidad de vida de los adultos mayores, en términos de alimentación y la salud de las personas mayores a pesar del confinamiento tal como se manifiesta en los relatos descritos en el apartado resultados.

Por otro lado, podemos destacar que la concienciación de la población sobre los factores que permean las condiciones de la salud biopsicosocial de los adultos mayores, puede conducir a un proceso educativo positivo en la lucha contra la estigmatización que denigra su identidad y representatividad en la sociedad. Así, para contrarrestar la percepción errónea de que el envejecimiento es sinónimo de enfermedad, consideramos que la longevidad representa un ciclo de pérdidas progresivas de la naturaleza humana. Sin embargo, mientras esta pandemia persiste, las poblaciones vulnerables, especialmente los adultos mayores, denotan y carecen de reconocimiento por parte de los organismos públicos competentes, además del impacto negativo causado por la limitación en la comunicación interpersonal, dificultando el proceso de socialización.

\section{Limitaciones y proyecciones del estudio}

Entre las limitaciones presentadas en el estudio, está la necesidad de aumentar el tamaño de la muestra, ya que los participantes pertenecen sólo a los programas que desarrolla el Instituto Nacional de Deportes de Chile. Otra limitación relevante, es la imposibilidad de acompañamiento de los sujetos en los momentos de 
realización de sus sesiones en los talleres, producto de la pandemia Covid-19. En términos de proyecciones, el estudio aporta antecedentes útiles, para su contrastación con investigaciones en diferentes contextos internacionales, a objeto de comprender como las personas mayores han vivido la pandemia Covid-19 y su impacto en la salud y el ejercicio físico, lo que conducirá seguramente al establecimiento de políticas de desarrollo de largo plazo, mejorando sustancialmente la calidad de vida de la sociedad adulta.

\section{Referencias}

Alcañiz, R. N., \& González-Moro, I. M. (2020). Valoración del grado de deterioro funcional y fragilidad en adultos mayores activos. Retos: nuevas tendencias en educación física, deporte y recreación, (38), 576-581. https://doi.org/ 10.47197/retos.v38i38.78252

Anton, S. D., Woods, A. J., Ashizawa,T., Barb, D., Buford,T.W., Carter, C. \& Pahor, M. (2015). Successful aging: advancing the science of physical independence in older adults. Ageing Research Reviews, 24, 304-327. https://doi.org/ 10.1016/j.arr.2015.09.005

Applegate, W. B., \& Ouslander, J. G. (2020). COVID 19 presents high risk to older persons. Journal of the American Geriatrics Society, 68(4), 681. doi: 10.1111/jgs. 16426

Ayalon, L., Chasteen, A., Diehl, M., Levy, B., Neupert, S. D., Rothermund, K., \&Wahl, H.W. (2020). Aging in times of the COVID-19 pandemic: Avoiding ageism and fostering intergenerational solidarity. The Journals of Gerontology: Series $B$. https://doi.org/10.1093/geronb/gbaa051

Armitage, R., \& Nellums, L. B. (2020). COVID-19 and the consequences of isolating the elderly. The Lancet Public Health, 5(5), e256. https://doi.org/10.1016/S24682667(20)30061-X

Beard, J. R., Officer, A., De Carvalho, I. A., Sadana, R., Pot,A. M., Michel, J. P., \& Thiyagarajan, J. A. (2016). TheWorld report on ageing and health: a policy framework for healthy ageing. The Lancet, 387(10033), 2145-2154. https:// doi.org/10.1016/S0140-6736(15)00516-4

Berg-Weger, M., \& Morley, J. E. (2020). Loneliness and social isolation in older adults during the Covid-19 pandemic: Implications for gerontological social work. J Nutr Health Aging. 24(5):456-458. https://doi.org/10.1007/ s12603-020-1366-8

Bengtsson M. (2016) How to plan and perform a qualitative study using content analysis. NursingPlus Open, 2: 8-14. https://doi.org/10.1016/j.npls.2016.01.001

Bonanad, C., García-Blas, S., Tarazona-Santabalbina, F. J., DíezVillanueva, P., Ayesta, A., Forés, J. S., \& Martínez-Sellés, M. (2020). Coronavirus: la emergencia geriátrica de 2020. Documento conjunto de la Sección de Cardiología Geriátrica de la Sociedad Española de Cardiología y la
Sociedad Española de Geriatría y Gerontología. Revista Española de Cardiología. 73(7) 569-576. https://doi.org/ 10.1016/j.recesp.2020.03.027

Bonsdorff M, \& RantanenT. (2011). Progression of functional limitations in relation to physical activity: a life course approach. European Review of Aging and Physical Activity, 8:23-30. https://doi.org/10.1007/s11556-010$0070-9$

Brooke, J., \& Jackson, D. (2020). Older people and COVID 19: Isolation, risk and ageism. Journal of Clinical Nursing. https://doi.org/10.1111/jocn.15274

Chou, C. H., Hwang, C. L., \&Wu,Y.T. (2012). Effect of exercise on physical function, daily living activities, and quality of life in the frail older adults: A meta-analysis. Archives of Physical Medicine and Rehabilitation, 93(2), 237-244. https://doi.org/10.1016/j.apmr.2011.08.042

Cuello-Carballo, M. B., Díaz-Alfonso, H., Cruz-Quesada, J. E., Carbó-Rodríquez, H. L., \& Dopico-Ravelo, D. (2020). Caracterización clínico-epidemiológica de los pacientes confirmados con la COVID-19 en Pinar del Río. Revista de Ciencias Médicas de Pinar del Río, 24(5). http:// scielo.sld.cu/scielo.php?pid=S1561$31942020000500005 \&$ script $=$ sci_arttext\&tlng $=$ pt

Crespo, R. M., \& Crespo, M. M. (2020). Pandemia COVID19, la nueva emergencia sanitaria de preocupación internacional: una revisión. Medicina de Familia. SEMERGEN. https://doi.org/10.1016/j.semerg.2020.05.010

Conde-Sala, J. L., Portellano-Ortiz, C., Calvó-Perxas, L., \& Garre-Olmo, J. (2017). Quality of life in people aged $65+$ in Europe: associated factors and models of social welfare - analysis of data from the SHARE project (Wave 5). Quality of life Research, 26(4), 1059-1070. https:// doi.org/10.1007/s11136-016-1436-x

Condeza, A. R., Bastias, G., Valdivia, G., Cheix, C., Barrios, X., Rojas, R., \& Fernández, F. (2016). Adultos mayores en Chile: descripción de sus necesidades en comunicación en salud preventiva. Cuadernos. info, (38), 85-104. http:// dx.doi.org/10.7764/cdi.38.964

De Carvalho, D. A., Brito, A. F., Dos Santos, M. A. P., de Siqueira Nogueira, F. R., de Moura Sá, G. G., de Oliveira Neto, J. G., \& dos Santos, E. P. (2017). Prevalence of physical exercise in the elderly and its relationship with difficulties and lack of specific professional advice. Revista Brasileira de Ciência e Movimento, 25(1), 29-40. http:// dx.doi.org/10.18511/rbcm.v25i1.6467

De Oliveira, J. C., Vinhas, W., \& Rabello, L. G. (2020). Benefícios do exercício físico regular para idosos. Brazilian Journal of Development, 6(3), 15496-15504. DOI: $10.34117 /$ bjdv6n3-429

De Souto Barreto, P., Morley, J. E., Chodzko-Zajko,W., Pitkala, K. H., Weening-Djiksterhuis, E., Rodriguez-Manas, L., \& Network, G. (2016). Recommendations on physical activity and exercise for older adults living in long-term care facilities: a taskforce report. Journal of the American 
Medical Directors Association, 17(5), 381-392. https:// doi.org/10.1016/j.jamda.2016.01.021

Dunsky, A. (2019). The effect of balance and coordination exercises on quality of life in older adults: a mini-review. Frontiers in Aging Neuroscience, 11, 318. doi: 10.3389/ fnagi.2019.00318

Farajzadeh M, Gheshlagh R, Sayehmiri K. (2017). Health related quality of life in Iranian elderly citizens: a systematic review and meta-analysis. International Journal of Community Based Nursing and Midwifery, 5(2): 100-111. PMC5385233

Faustino, A. M., \& Neves, R. (2020). Benefícios da prática de atividade física em pessoas idosas: revisão de literatura. Revista Eletrônica Acervo Saúde, 12(5), e3012-e3012. https:/ / doi.org/10.25248/reas.e3012.2020

Fernández-Ballesteros, R. (2011). Quality of Life in old age. Problematic issues. Applied Research Quality of Life, 10:2140. https: / / doi.org/10.1007/s11482-010-9110-x

Flett, G. L., \& Heisel, M. J. (2020). Aging and feeling valued versus expendable during the COVID-19 pandemic and beyond: A review and commentary of why mattering is fundamental to the health and well-being of older adults. International Journal of Mental Health and Addiction, 1-27. https: / / doi.org/10.1007/s11469-020-00339-4

Flick, U. (2018). An introduction to qualitative research. Sage Publications Limited.

Flores R. (2009). Observando observadores: una introducción a las técnicas cualitativas de investigación social. Santiago: Ediciones UC.

Forte, R., Boreham, C. A., De Vito, G., \& Pesce, C. (2015). Health and quality of life perception in older adults: the joint role of cognitive efficiency and functional mobility. International Journal of Environmental Research and Public Health, 12(9), 11328-11344. https://doi.org/10.3390/ ijerph120911328

Froment, F., \& González, A. J. G. (2018). Beneficios de la actividad física sobre la autoestima y la calidad de vida de personas mayores. Retos: nuevas tendencias en educación física, deporte y recreación, (33), 3-9.

Garcia-Molina,A. Carbonell-Baeza,A., \& Delgado-Fernández, M. (2010). Health benefits of physical activity in older people. Revista Internacional de Medicina y Ciencias de La Actividad Fisica y Del Deporte, 10(40), 556-576. http:// cdeporte.rediris.es/revista/re.

Gibbs, G. (2007). Analyzing qualitative data. Los Angeles, CA: SAGE Publications. http://dx.doi.org/10.4135/ 9781849208574

Given, L. M. (Ed.). (2008). The Sage encyclopedia of qualitative research methods. Thousand Oaks, CA: SAGE Publications

Graneheim, U. H., Lindgren, B. M., \& Lundman, B. (2017). Methodological challenges in qualitative content analysis: A discussion paper. Nurse EducationToday, 56, 29-34. https: / /doi.org/10.1016/j.nedt.2017.06.002

Grossman, E. S., Hoffman,Y. S., Palgi,Y., \& Shrira, A. (2020). COVID-19 related loneliness and sleep problems in older adults: Worries and resilience as potential moderators. Personality and individual differences, 168, 110371. https:/ /doi.org/10.1016/j.paid.2020.110371

Hale D, Marshall K. (2017). Physical activity for older adults. Home Healthcare Now, 35(3):172-173. doi: 10.1097/ NHH.0000000000000496.

Heredia, N. M., Rodríguez, E. S., \& García,A. M. R. (2021). Beneficios de la actividad física para la promoción de un envejecimiento activo en personas mayores: revisión bibliográfica. Retos: nuevas tendencias en educación física, deporte y recreación, (39), 12. http: / / hdl.handle.net/10481/ 64030

Huxhold O, Miche M, Schüz B. (2013). Benefits of having friends in older ages: differential effects of informal social activities on well-being in middle-aged and older adults.J Gerontol B Psychol Sci Soc Sci, 69(3):366-75. https:// doi.org/10.1093/geronb/gbt029

INE (2018). Censo población y vivienda. Instituto Nacional de Estadística, Chile.

Issa R, Kilpi F,Webber L. (2016). Physical activity and public health: Updated recommendation for adults from the American college of sports medicine and the American heart association. Pakistan Journal of Nutrition, 17(2):10781086. doi: 10.1249/mss.0b013e3180616b27

Li, C. I., Lin, C. H., Lin, W.Y., Liu, C. S., Chang, C. K., Meng, N. H., \& Lin, C. C. (2014). Successful aging defined by healthrelated quality of life and its determinants in communitydwelling elders. BMC Public Health, 14(1), 1013. https: / / doi.org/10.1186/1471-2458-14-1013

Losada-Baltar, A., Jiménez-Gonzalo, L., Gallego-Alberto, L., Pedroso-Chaparro, M. D. S., Fernandes-Pires, J., \& Márquez-González, M. (2020). «We're staying at home». Association of self-perceptions of aging, personal and family resources and loneliness with psychological distress during the lock-down period of COVID-19. The Journals of Gerontology: Series B. https://doi.org/10.1093/ geronb/gbaa048

Machón, M., Larrañaga, I., Dorronsoro, M., Vrotsou, K., \& Vergara, I. (2017). Health-related quality of life and associated factors in functionally independent older people. BMC Geriatrics, 17(1), 19. https://doi.org/ 10.1186/s12877-016-0410-3

Maguiña-Vargas, C., Gastelo Acosta, R., \&Tequen Bernilla,A. (2020). El nuevo Coronavirus y la pandemia del Covid19. Revista Médica Herediana, 31(2), 125-131. http:// dx.doi.org/10.20453/rmh.v31i2.3776

Moreira, A., \& Costa, A. P. (2016). Introduction: Qualitative Analysis: Quantifying Quality and Qualifying Quantity. The Qualitative Report,21(13), 1-5. fromhttps:// nsuworks.nova.edu/tqr/vol21/iss 13/1

Morse J. (2015). Critical analysis of strategies for determining rigor in qualitative inquiry. Qualitative Health Research, 25(9):1212-1222. https://doi.org/10.1177/ 1049732315588501 
Netuveli, G., \& Blane, D. (2008). Quality of life in older ages. British Medical Bulletin, 85(1), 113-126. https: / / doi.org/ $10.1093 / \mathrm{bmb} / \mathrm{ldn} 003$

Ornell, F. Schuch, J. B., Sordi, A. O., \& Kessler, F. H. (2020). Pandemia de medo e Covid-19: impacto na saúde mental e possíveis estratégias. Revista Debates in Psychiatry, 2-7.

Pinazo-Hernandis, S. (2020). Impacto psicosocial de la COVID-19 en las personas mayores: problemas y retos. Revista Española de Geriatría y Gerontología, 55(5), 249252. https://doi.org/10.1016/j.regg.2020.05.006

Ping,W., Zheng, J., Niu, X., Guo, C., Zhang, J., Yang, H., \& Shi, Y. (2020). Evaluation of health-related quality of life using EQ-5D in China during the COVID-19 pandemic. PloS one, 15(6), e0234850. https://doi.org/10.1371/ journal.pone. 0234850

PérezAbreu, M. R., GómezTejeda, J. J., \& Dieguez Guach, R. A. (2020). Características clínico-epidemiológicas de la COVID-19. Revista Habanera de Ciencias Médicas, 19(2). h t t p : / / s c i e l o.s l d. c u / scielo.php?script $=$ sci_arttext $\&$ pid $=$ S 1729 . 519X2020000200005

Rahman, A., \& Jahan, Y. (2020). Defining a 'Risk Group'and Ageism in the Era of COVID-19. Journal of Loss and Trauma, 1-4. https://doi.org/10.1080/ 15325024.2020 .1757993

Ruaro, M. F., Santana, J. O., Gusmão, N., De França, E., Carvalho, B. N., Farinazo, K. B., \& Caperuto, É. C. (2019). Effects of strength training with and without blood flow restriction on quality of life in the elderly. Journal of Physical Education and Sport, 19, 787-794. DOI:10.7752/ jpes.2019.s3112

Russo, M. J., Kañevsky, A., Leis, A., Iturry, M., Roncoroni, M., Serrano, C., ... \& Zuin, D. (2020). Papel de la actividad física en la prevención de deterioro cognitivo y demencia en adultos mayores: una revisión sistemática. Neurología Argentina, 12(2), 124-137. https://doi.org/ 10.1016/j.neuarg.2020.01.003

Sannicandro, I., Cofano, G., Rosa, R.A., \& Colella, D. (2020). The sedentary contrast strategies in the older people during the spread of Covid 19: The Italian experience. Sport Science, 14 (1): 60-64. http://sposci.com/PDFS/ BR1401/04\%20CL\%2010\%20IS.pdf

Silverman, D. (2014). Interpreting qualitative data (5th ed.). Los Angeles, CA: SAGE Publications.

Tavares, R. E., Jesus, M. C. P., Machado, D. R., Braga,V.A. S., Tocantins, F. R., \& Merighi, M. A. B. (2017). Envelhecimento saudável na perspectiva de idosos: Uma revisão integrativa. Rev Bras Geriatr Gerontol, 20(6), 878889. DOI: 10.1590/1981-22562017020.170091

Teixeira-Salmela L. (2016). Effects of physical and therapeutic activities on mature and elderly adults. Fisioterapia Brasil, 2(2):99-106. http:/ / dx.doi.org/10.33233/fb.v2i2.628

Torres, Á. F. R., Gaibor, J.A. G., \& Pozo, D. I. L. (2020). Los beneficios de la actividad física en la calidad de vida de los adultos mayores. EmásF: Revista Digital de Educación Físi- ca, (63), 22-35. file:///C:/Users/10870516/ Downloads/Dialnet- -7279808.pdf

Tyndall A, Clark C, Anderson T. (2018). Protective effects of exercise on cognition and brain health in older adults. Exercise and Sport Sciences Reviews, 46(4): 215-223. doi: 10.1249/JES.0000000000000161

Vaismoradi M, Turunen H, Bondas T. (2013). Content analysis and thematic analysis: Implications for conducting a qualitative descriptive study. Nursing \& Health Sciences, 15(3): 398-405. https://doi.org/10.1111/nhs.12048

VanTilburg, T. G., Steinmetz, S., Stolte, E., van der Roest, H., \& de Vries, D. H. (2020). Loneliness and mental health during the COVID-19 pandemic: A study among Dutch older adults. The Journals of Gerontology: Series B. https: / / doi.org/10.1093/geronb/gbaa1 11

Vanleerberghe, P., DeWitte, N., Claes, C., Schalock, R. L., \& Verté, D. (2017). The quality of life of older people aging in place: a literature review. Quality of Life Research, 26(11), 2899-2907. https: / /doi.org/10.1007/s11136-0171651-0

Viana, S. A. A., de Lima Silva, M., \& de Lima, P. T. (2020). Impacto na saúde mental do idoso durante o período de isolamento social em virtude da disseminação da doença COVID-19: uma revisão literária. Diálogos Em Saúde, 3(1). https: / / periodicos.iesp.edu.br/index.php/ dialogosemsaude/article/view/272/232

Vicentini de Oliveira, D., Magnani Branco, B. H., Costa de Jesus, M., Sepúlveda-Loyola, W., Gonzáles-Caro, H., Morais Freire, G. L., ... \& Nascimento Júnior, J. R. (2021). Relación entre la actividad física vigorosa y la composición corporal en adultos mayores. Nutrición Hospitalaria, 38(1), 60-66. https:/ /dx.doi.org/10.20960/nh.03254.

Viladrosa, M., Casanova, C., Ghiorghies, A. C., \& Jürschik, P. (2017). El ejercicio físico y su efectividad sobre la condición física en personas mayores frágiles. Revisión sistemática de ensayos clínicos aleatorizados. Revista Española de Geriatría y Gerontología, 52(6), 332-341. https: / / doi.org / 10.1016/j.regg.2017.05.009

Villarreal-Angeles, M. A., Moncada-Jimenez, J., \& Ruiz-Juan, F. (2021). Mejora de variables psicológicas en Adultos Mayores mediante Pilates. Retos: nuevas tendencias en educación física, deporte y recreación, (40), 47-52. https:// doi.org/10.47197/retos.v1i40.74307

Whitehead B, \& Blaxton J. (2017). Daily Well-Being Benefits of Physical Activity in Older Adults: Does Time or Type Matter? Gerontologist, 57(6):1062-1071. https:// doi.org/10.1093/geront/gnw250

Williams, G., \& Cañon-Montañez, W. (2020). COVID-19: What we've learned so far. Revista Cuidarte, 11(2). https: / /doi.org/10.15649/cuidarte.1225

World Health Organization. (2020). Novel Coronavirus (2019-nCoV) technical guidance.

World Health Organization. (2015). World report on ageing and health. 